Page 1 of 20

University of Maryland Computer Science CS-TR-5028

University of Maryland Institite for Advanced Computer Studies TR-2013-05

\title{
Efficient iterative algorithms for linear stability analysis of incompressible flows
}

\author{
HOWARD C. ELMAN \\ Department of Computer Science and Institute for Advanced Computer Studies \\ University of Maryland, College Park, MD 20742, USA \\ elman@cs.umd.edu \\ AND \\ MinghaO W. Rostami \\ Department of Mathematical Sciences \\ Worcester Polytechnic Institute, Worcester, MA 01609, USA \\ mwu@wpi,edu
}

\begin{abstract}
Linear stability analysis of a dynamical system entails finding the rightmost eigenvalue for a series of eigenvalue problems. For large-scale systems, it is known that conventional iterative eigenvalue solvers are not reliable for computing this eigenvalue. A more robust method recently developed in Elman \& Wu (2012) and Meerbergen \& Spence (2010), Lyapunov inverse iteraiton, involves solving large-scale Lyapunov equations, which in turn requires the solution of large, sparse linear systems analogous to those arising from solving the underlying partial differential equations. This study explores the efficient implementation of Lyapunov inverse iteration when it is used for linear stability analysis of incompressible flows. Efficiencies are obtained from effective solution strategies for the Lyapunov equations and for the underlying partial differential equations. Existing solution strategies are tested and compared, and a modified version of a Lyapunov solver is proposed that achieves significant savings in computational cost.
\end{abstract}

\section{Introduction}

In this paper, we will discuss efficient computational algorithms for linear stability analysis of a large-scale dynamical system of the form

$$
M u_{t}=f(u, \alpha),
$$

where $\mathbf{M} \in \mathbb{R}^{n \times n}$ is called the mass matrix and is large and sparse, $u \in \mathbb{R}^{n}$ is the state variable (velocity, pressure, temperature, etc.), and $\alpha$ is a physical parameter. Such a dynamical system arises from spatial discretization of two- or three-dimensional partial differential equations (PDEs).

Linear stability analysis is a standard approach to studying the sensitivity of a steady state $\bar{u}$ of (1.1) to small perturbations: roughly speaking, if all small perturbations introduced to $\bar{u}$ will eventually die out, then $\bar{u}$ is stable; and if some of them will grow with time, then $\bar{u}$ is considered unstable. We are especially interested in identifying the critical point $\left(\bar{u}_{c}, \alpha_{c}\right)$ at which $\bar{u}$ changes from being stable to unstable. The linear stability of $\bar{u}$ is determined by the rightmost eigenvalue (i.e., the eigenvalue with algebraically largest real part) of a generalized eigenvalue problem

$$
J(\bar{u}, \alpha) x=\mu \mathbf{M} x,
$$

where $J(\bar{u}, \alpha)=\frac{\partial f}{\partial u}(\bar{u}, \alpha)$ is the Jacobian matrix, which is often large, sparse, and in general, nonsymmetric. 
If the rightmost eigenvalue of (1.2) has negative real part, then $\bar{u}$ is stable; otherwise, it is unstable. At the critical point $\left(\bar{u}_{c}, \alpha_{c}\right)$, the rightmost eigenvalue of (1.2) has real part zero.

Consequently, $\left(\bar{u}_{c}, \alpha_{c}\right)$ can be located by monitoring the rightmost eigenvalue of (1.2) along a path of stable steady states. Commonly used iterative eigenvalue solvers such as Arnoldi's method and its variants (see Stewart (2001)) work well when a small set of eigenvalues of (1.2) near a given point $\sigma \in \mathbb{C}$ (called the "shift") are sought. Thus, a good estimate for the rightmost eigenvalue of (1.2) would be an ideal choice for $\sigma$. Unfortunately, such an estimate is usually not available. In practice, it is common to choose $\sigma=0$, that is, to compute a number of eigenvalues of (1.2) closest to zero hoping that the rightmost one is among them. One major disadvantage of this strategy lies in its lack of robustness: a rightmost eigenvalue with large imaginary part may not be found.

Recently, a more robust method for computing the rightmost eigenvalue of (1.2) has been developed in Elman \& Wu (2012). This method finds the rightmost eigenvalue of (1.2) by introducing a new eigenvalue problem in the form of a Lyapunov equation and computing its eigenvalue with smallest modulus. A brief description of this method is as follows. Let $\mathbf{A}=J\left(\bar{u}_{0}, \alpha_{0}\right)$ where $\left(\bar{u}_{0}, \alpha_{0}\right)$ is any point in the stable regime. In addition, let $\mu_{1}$ denote the rightmost eigenvalue of $\mathbf{A} x=\mu \mathbf{M} x$ and $x_{1}$ (with $\|x\|_{2}=1$ ) the eigenvector associated with it. It was shown in Elman \& Wu (2012) and Meerbergen \& Vandebril (2012) that if $\mathbf{M}$ is nonsingular, then the eigenvalue with smallest modulus of

$$
S Z+Z S^{\top}+\lambda\left(2 S Z S^{\top}\right)=0
$$

where $\mathbf{S}=\mathbf{A}^{-1} \mathbf{M}$ is $-\frac{1}{2}\left(\mu_{1}+\overline{\mu_{1}}\right)$. Moreover, under the generic assumptions that $\mu_{1}$ is simple and that except for $\overline{\mu_{1}}$, no other eigenvalue of $\mathbf{A} x=\mu \mathbf{M} \times$ has the same real part as $\mu_{1}$, there is a unique (up to a scalar multiplier), real and symmetric "eigenvector" of (1.3) associated with $-\frac{1}{2}\left(\mu_{1}+\overline{\mu_{1}}\right)$ given by $x_{1} x_{1}^{*}+\overline{x_{1}} x_{1}^{\top}$. This eigenvector is of rank 1 if $\mu_{1}$ is real or rank 2 otherwise. As a result, it can be represented efficiently using its truncated eigenvalue decomposition $\mathcal{V D} \mathcal{V}^{\top}$, where the matrix $\mathcal{V}$ consists of one or two orthonormal columns that form a basis for $\operatorname{span}\left\{x_{1}, \overline{x_{1}}\right\}$.

The observation above suggests the following approach to finding the rightmost eigenvalue of $\mathbf{A} x=$ $\mu \mathbf{M} x$ : first solve (1.3) for its eigenvalue with smallest modulus and the associated eigenvector, and then solve the small eigenvalue problem $\left(\mathcal{V}^{\top} \mathbf{A} \mathcal{V}\right) \mathbf{y}=\mu\left(\mathcal{V}^{\top} \mathbf{M} \mathcal{V}\right) \mathrm{y}$. The advantage of this strategy is that unlike for the rightmost eigenvalue, there are many robust methods for computing an eigenvalue with smallest modulus, in particular, inverse iteration (also known as inverse power method, see Stewart (2001)). In Elman \& Wu (2012), a variant of inverse iteration referred to as Lyapunov inverse iteration was used. This algorithm was first proposed in Meerbergen \& Spence (2010) for eigenvalue problems similar in structure to (1.3); the methodology was developed to take advantage of the special low-rank structure of the "eigenvector" $Z$ of (1.3).

Applying Lyapunov inverse iteration to (1.3) requires solving a large-scale Lyapunov equation

$$
\mathrm{SY}+\mathrm{YS}^{\top}=\mathrm{PCP}^{\top}
$$

at each step, where $P \in \mathbb{R}^{n \times r}$ with $r=1$ or 2 (see Elman \& Wu (2012)). Hence, the implementation of Lyapunov inverse iteration depends on solving (1.4) efficiently. The solution to (1.4) often has low-rank approximation (see Antoulas et al. (2001); Grasedyck (2004); Kressner \& Tobler (2010); Penzl (2000)), and iterative methods for computing such an approximation (see, for instance, Druskin \& Simoncini (2011); Saad (1990); Simoncini (2007)) entail matrix-vector products with matrices that are rational functions of $\mathrm{S}$, which in turn require solving large, sparse linear systems. For large-scale discretization of the PDEs, practical implementations entail the use of preconditioned iterative methods for performing these solves. Our aim in this work is to explore the effectiveness of several solution algorithms for the linear systems 
arising from Lyapunov inverse iteration, and, more importantly, to develop a way to reduce the costs of these solves.

The rest of this paper is organized as follows. In section 2, we review two iterative Lyapunov solvers: the standard Krylov subspace method developed in Saad (1990) and the rational Krylov subspace method developed in Druskin \& Simoncini (2011), both of which have been used in Elman \& Wu (2012). In particular, we introduce the types of linear systems that need to be solved in the implementation of these two methods. In section 3, we first review and test the iterative methods developed for these systems arising from incompressible Navier-Stokes equations. Then we incorporate these methods into the Lyapunov solvers, which are tested on several examples considered. Based on the numerical results, we propose in section 4 a modified version of the rational Krylov subspace method and demonstrate that it achieves significant savings in computational cost. In section 5, justification for this modification is provided. Some concluding remarks are given in section 6 .

\section{Review of iterative Lyapunov solvers}

In this section, we review two Lyapunov solvers that can be used for Lyapunov inverse iteration, see Elman \& Wu (2012): the standard Krylov subspace method (Jaimoukha \& Kasenally (1994); Saad (1990)) and the rational Krylov subspace method (RKSM) (Druskin et al. (2011); Druskin \& Simoncini (2011)). Both methods construct a low-rank approximate solution to (1.4) of the form $V_{m} X_{m} V_{m}^{\top}$, where the columns of $V_{m}$ form an orthonormal basis for a small subspace of $\mathbb{R}^{n}$ and $X_{m}$ is the solution to a small Lyapunov equation that can be solved using direct methods (Bartels \& Stewart (1972); Hammarling (1982)). Let the residual associated with (1.4) be

$$
R=S\left(V_{m} X_{m} V_{m}^{\top}\right)+\left(V_{m} X_{m} V_{m}^{\top}\right) S^{\top}-P C P^{\top}
$$

The small Lyapunov equation is obtained by imposing a Galerkin condition of the form trace $\left(R Z^{\top}\right)=0$, where $Z$ is any matrix of the form $V_{m} Q V_{m}^{\top}$.

In Elman \& Wu (2012), a real, symmetric and rank-1 matrix $Z^{(0)}=v_{0} v_{0}^{\top}$ was chosen to be the starting guess of the target eigenvector of (1.3) where $v_{0}$ is a random vector with unit norm in $\mathbb{R}^{n}$. As a result, the right-hand side of the Lyapunov equation (1.4) that needs to be solved in the first step of Lyapunov inverse iteration is $-2 S Z^{(0)} S^{\top}=-2 S v_{0} v_{0}^{\top} S^{\top}$, i.e., $\mathrm{P}$ is a single vector with unit norm. As the algorithm proceeds, in subsequent iterations, the rank of the right-hand side of (1.4) may change to 2, in which case $P$ will have two orthonormal columns. For simplicity, in the rest of this paper, we focus on the solution of the first Lyapunov equation, which has a rank-1 right-hand side. It is straightforward to generalize the algorithms presented here to the case where P has multiple columns.

In the standard Krylov subspace method, a Krylov subspace that we are familiar with,

$$
\mathcal{K}_{\mathrm{m}}(\mathrm{S}, \mathrm{P})=\operatorname{span}\left\{\mathrm{P}, \mathrm{SP}, \mathrm{S}^{2} \mathrm{P}, \ldots, \mathrm{S}^{\mathrm{m}-1} \mathrm{P}\right\},
$$

is built. This method is outlined in Algorithm 2.1.

Algorithm 2.1 The standard Krylov subspace method for (1.4)

1. Given a tolerance $\tau$. Let $v_{1}=V_{1}=P$.

2. For $m=1,2, \cdots$

$$
\begin{array}{r}
\text { 2.1. } w=S v_{m} . \\
\text { For } i=1, \ldots, m \\
h_{i, m} \leftarrow v_{i}^{\top} w ;
\end{array}
$$




$$
w \leftarrow w-v_{i} h_{i, m}
$$

2.2. Solve the small Lyapunov equation

$$
H_{m} X_{m}+X_{m} H_{m}^{\top}=\left(V_{m}^{\top} P\right) C\left(V_{m}^{\top} P\right)^{\top}
$$

where $\mathrm{H}_{\mathrm{m}}=\mathrm{V}_{\mathrm{m}}^{\top} \mathrm{S} \mathrm{V}_{\mathrm{m}}$.

2.3. Compute the reduced $Q R$ factorization of $w: w=v_{m+1} h_{m+1, m}$.

2.4. If the residual norm $\|\mathrm{R}\|_{\mathrm{F}}<\tau$, then stop.

2.5. Else, $V_{\mathrm{m}+1} \leftarrow\left[\mathrm{V}_{\mathrm{m}}, v_{\mathrm{m}+1}\right]$.

In Algorithm 2.1, the matrix $\mathrm{H}_{m}=V_{m}{ }^{\top} S V_{m}$ is available at no cost since it is simply the upper Hessenberg matrix $\left[h_{i, j}\right]_{i, j=1}^{m}$. As shown in Jaimoukha \& Kasenally (1994), the residual norm $\|R\|_{F}$ of (1.4) can be computed cheaply as well. At each step of Algorithm 2.1, the matrix-vector product $S v_{m}$ is formed. Since $S=\mathbf{A}^{-1} \mathbf{M}$ where $\mathbf{A}$ is the Jacobian matrix, computing $S v_{m}$ entails one solve of the linear system

$$
\mathbf{A} x=b
$$

where $\mathrm{b}=\mathbf{M} v_{\mathrm{m}}$. This is precisely the kind of linear system that needs to be solved in the computation of the steady-state solution for (1.1).

The rational Krylov subspace method (RKSM) was originally developed in Ruhe $(1984,1994)$ for the computation of the interior eigenvalues of $S$. This method constructs a subspace

$$
\mathcal{K}_{m}(S, P, s)=\operatorname{span}\left\{P,\left(S-s_{1} I\right)^{-1} P,\left(S-s_{2} I\right)^{-1}\left(S-s_{1} I\right)^{-1} P, \ldots, \prod_{j=1}^{m-1}\left(S-s_{m-j} I\right)^{-1} P\right\},
$$

where $\mathbf{s}=\left\{s_{j}\right\}_{j=1}^{m-1} \in \mathbb{C}^{m-1}$ is a set of shifts that need to be selected by some means. The utility of this method for solving large-scale Lyapunov equations has recently been investigated in Druskin \& Simoncini (2011). An algorithmic description reads as follows:

Algorithm 2.2 The rational Krylov subspace method for (1.4)

1. Given a tolerance $\tau$ and a shift $s_{1}$. Let $v_{1}=V_{1}=P$.

2. For $m=1,2, \cdots$

2.1. $w=\left(\mathrm{S}-s_{\mathrm{m}} \mathrm{I}\right)^{-1} v_{\mathrm{m}}$.

For $i=1, \ldots, m$

$h_{i, m} \leftarrow v_{i}^{\top} w$;

$w \leftarrow w-v_{i} h_{i, m}$.

2.2. Compute the reduced $Q R$ factorization of $w: w=v_{m+1} h_{m+1, m}$.

2.3. Compute $T_{m}=V_{m}^{T} S V_{m}$ and solve the small Lyapunov equation

$$
T_{m} X_{m}+X_{m} T_{m}^{\top}=\left(V_{m}^{\top} P\right) C\left(V_{m}^{\top} P\right)^{\top} .
$$

2.4. If $\|R\|_{F}<\tau$, then stop.

2.5. Else, $\mathrm{V}_{\mathrm{m}+1} \leftarrow\left[\mathrm{V}_{\mathrm{m}}, v_{\mathrm{m}+1}\right]$ and compute the next shift $\mathrm{s}_{\mathrm{m}+1}$.

In Druskin et al. (2010) (for symmetric S) and Druskin \& Simoncini (2011) (for general S), adaptive and parameter-free approaches for generating the shifts $\mathbf{s}$ were proposed. Both approaches require some knowledge of the spectrum of S. In Druskin \& Simoncini (2011), the first shift $s_{1}$ is chosen to be a rough 
estimate of either $-\operatorname{Re}_{\min }(\theta)$ or $-\operatorname{Re}_{\max }(\theta)$, where $\operatorname{Re} e_{\min }(\theta)$ and $\operatorname{Re}_{\max }(\theta)$ denote the minimum and maximum real parts of the eigenvalues of $S$, respectively. (Since we assume $\mathbf{A}$ to be the Jacobian matrix evaluated at any stable point $\left(\bar{u}_{0}, \alpha_{0}\right)$, the eigenvalues of $S$ all lie in the left half of the complex plane, i.e., $0>\operatorname{Re}_{\max }(\theta)>\operatorname{Re} e_{\min }(\theta)$.) Let $\mathscr{I}=\left[-\operatorname{Re}_{\max }(\theta),-\operatorname{Re} e_{\min }(\theta)\right]$ and $\left\{\widehat{\theta}_{j}\right\}_{j=1}^{m}$ denote the eigenvalues of $\mathrm{T}_{\mathrm{m}}$, which will be updated at each iteration of Algorithm 2.2 and reflect the most recent information on the spectrum of $S$. Each subsequent shift $s_{m+1}$ is then chosen as follows:

$$
s_{m+1}=\arg \left(\max _{s \in \mathscr{I}} \frac{1}{\left|r_{m}(s)\right|}\right), \text { where } r_{m}(s)=\frac{\prod_{j=1}^{m}\left(s-\widehat{\theta}_{j}\right)}{\prod_{j=1}^{m}\left(s-s_{j}\right)} .
$$

Once $\left\{\widehat{\theta}_{j}\right\}_{j=1}^{m}$ are known, this selection process only involves sampling the rational function $r_{m}(s)$ on the interval $\mathscr{I}$, which is cheap. By Proposition 4.2 of Druskin \& Simoncini (2011), the residual norm $\|\mathrm{R}\|_{\mathrm{F}}$ of this method is also easy to compute.

We now look into the linear systems that arise from Algorithm 2.2 when it is applied to (1.4). Since

$$
\left(\mathrm{S}-\mathrm{s}_{\mathrm{m}} \mathrm{I}\right)^{-1}=\left(\mathbf{A}^{-1} \mathbf{M}-s_{\mathrm{m}} \mathbf{A}^{-1} \mathbf{A}\right)^{-1}=\left(\mathbf{M}-s_{\mathrm{m}} \mathbf{A}\right)^{-1} \mathbf{A},
$$

computing $\left(S-s_{m} \mathrm{I}\right)^{-1} v_{m}$ entails the solution of a linear system of the form

$$
(\mathbf{M}-\mathrm{s} \mathbf{A}) \mathrm{x}=\mathrm{b}
$$

where $s>0$ and $\mathrm{b}=\mathbf{A} v_{m}$. The structure of (2.5) is exactly like that of the linear systems that need to be solved in the computation of a fully implicit iteration for a transient solution to (1.1), where s plays the role of the time step $\Delta t$.

Unlike in the standard Krylov subspace method, extra work is required to obtain the matrix $T_{m}=$ $V_{m}^{\top} S V_{m}$ in step 2 of Algorithm 2.2. Computing it naively requires $m$ matrix-vector products with $S$, i.e., $\mathrm{m}$ solves with A. A more efficient way of generating this matrix was proposed in Ruhe (1994) (see also Proposition 4.1 of Druskin \& Simoncini (2011)) which only requires knowing $S v_{m+1}$, or equivalently, one solve of (2.2) where $\mathrm{b}=\mathbf{M} v_{\mathrm{m}+1}$.

To sum up, when applied to (1.4), each iteration of the standard Krylov subspace method requires one solve of (2.2) in order to compute a new Krylov vector, whereas each iteration of RKSM requires one solve of (2.5) to compute a new Krylov vector and an additional solve of (2.2) to construct the matrix $T_{m}=V_{m}^{\top} S V_{m}$.

\section{Numerical results}

In the previous section, we have shown that solving the Lyapunov equation (1.4) arising from Lyapunov inverse iteration requires multiple solves of the linear systems (2.2) and/or (2.5), which are essentially the types of equations that arise in solving steady or transient PDEs. Therefore, iterative solution methods developed for such PDEs can be applied directly in Lyapunov inverse iteration. These strategies often involve designing efficient preconditioners that approximate the discrete versions of certain differential operators.

The dynamical system (1.1) that we consider in this study is the spatial discretization of the NavierStokes equations modeling incompressible flows,

$$
\begin{aligned}
u_{\mathrm{t}}-v \nabla^{2} \mathrm{u}+\mathrm{u} \cdot \nabla \mathrm{u}+\nabla \mathrm{p} & =0 \\
\nabla \cdot \mathrm{u} & =0
\end{aligned}
$$


6 of 20

subject to appropriate boundary conditions, where $u, p, v$ denote the velocity, pressure and kinematic viscosity, respectively. In order to examine the performance of Lyapunov solvers with iterative linear solves, we proceed in two steps: review some preconditioners developed for (3.1) and test them on (2.2) and (2.5), and then integrate these solution techniques into the Lyapunov solvers described in the previous section and apply them to (1.4) arising from Lyapunov inverse iteration.

\subsection{Iterative solves of the linear systems}

The matrices $\mathbf{A}$ and $\mathbf{M}$ arising from div-stable mixed finite element discretization of (3.1) have the block structure

$$
\mathbf{A}=\left[\begin{array}{cc}
F & B^{\top} \\
B & 0
\end{array}\right] \text { and } \mathbf{M}=\left[\begin{array}{cc}
-G & 0 \\
0 & 0
\end{array}\right] \text {. }
$$

The matrix blocks $F \in \mathbb{R}^{n_{\mathfrak{u}} \times n_{\mathfrak{u}}}, B \in \mathbb{R}^{n_{p} \times n_{\mathfrak{u}}}$ and $G \in \mathbb{R}^{n_{\mathfrak{u}} \times n_{\mathfrak{u}}}$ are all sparse, where $n_{\mathfrak{u}}+n_{p}=n_{\text {and }} n_{p}<$ $n_{u} . G$ is the velocity mass matrix, $B$ is the matrix representation of the discrete divergence operator, and $F$ resembles the matrix representation of the discrete convection-diffusion operator. (The precise definition of F can be found in Elman et al. (2005).) Since a nonsingular mass matrix is required (see Elman \& Wu (2012)), in the actual computation, we use instead a modified mass matrix

$$
\left[\begin{array}{cc}
-\mathrm{G} & \eta \mathrm{B}^{\mathrm{T}} \\
\eta \mathrm{B} & 0
\end{array}\right]
$$

where $\eta=-0.01$. The rightmost eigenvalue of $\mathbf{A} x=\mu \mathbf{M} x$ remain unchanged when this new mass matrix is used (see Cliffe et al. (1994)).

Preconditioners for (2.2) of the form

$$
\mathbf{P}=\left[\begin{array}{cc}
\mathrm{P}_{\mathrm{F}} & \mathrm{B}^{\mathrm{T}} \\
0 & -\mathrm{P}_{\mathrm{S}}
\end{array}\right]
$$

were developed in Elman et al. (2005) and Elman (2005), where $\mathrm{P}_{\mathrm{F}}$ is a preconditioner for $\mathrm{F}_{\text {and }} \mathrm{P}_{\mathrm{S}}$ is a preconditioner for the (dense) Schur complement $B F^{-1} B^{\top}$. In (3.3), we can choose $P_{F}$ to be $F$ itself and apply its inverse using a multigrid process (see Elman et al. (2005)). The main issue in designing $\mathbf{P}$ is the choice of $P_{S}$.

In Elman et al. (2005), two effective preconditioning strategies for the Schur complement were described: the pressure convection-diffusion preconditioner (PCD) and the least-squares commutator preconditioner (LSC). They are derived by approximately minimizing the discrete version of a commutator of certain differential operators using two different heuristics (see Elman et al. (2005)). The pressure convectiondiffusion preconditioner is defined to be

$$
A_{p} F_{p}^{-1} G_{p}
$$

where $A_{p}$ and $F_{p}$ are discrete Laplacian and convection-diffusion operators in the pressure space, respectively, and $G_{p}$ is the pressure mass matrix. The least-squares commutator preconditioner is defined to be

$$
\left(\mathrm{B} \widehat{\mathrm{G}}^{-1} \mathrm{~B}^{\top}\right)\left(\mathrm{B} \widehat{\mathrm{G}}^{-1} \mathrm{FG} \widehat{\mathrm{G}}^{-1} \mathrm{~B}^{\top}\right)^{-1}\left(\mathrm{~B} \widehat{\mathrm{G}}^{-1} \mathrm{~B}^{\top}\right)
$$

where $\widehat{G}$ is the diagonal matrix whose entries are taken from the diagonal of $G$.

The coefficient matrix $\mathbf{M}-\mathrm{sA}$ of (2.5) has the block structure

$$
\left[\begin{array}{cc}
-(s F+G) & (\eta-s) B^{\top} \\
(\eta-s) B & 0
\end{array}\right]
$$


similar to that of $\mathbf{A}$, and the pressure convection-diffusion preconditioner and the least-squares commutator preconditioner can be derived in an analogous manner for its Schur complement $-(\eta-s)^{2} B(s F+G)^{-1} B^{\top}$. They are

$$
-(\eta-s)^{2} A_{p}\left(s F_{p}+G_{p}\right)^{-1} G_{p}
$$

and

$$
-(\eta-s)^{2}\left(B \widehat{G}^{-1} B^{T}\right)\left(B \widehat{G}^{-1}(s F+G) \widehat{G}^{-1} B^{T}\right)^{-1}\left(B \widehat{G}^{-1} B^{T}\right),
$$

respectively. The shift $s$ in RKSM varies from one iteration to another. An important feature of both preconditioners is that they do not require any extra work to construct as $s$ changes.

We will explore both these preconditioning strategies, using improved variants of them developed to account for boundary effects; see Elman \& Tuminaro (2009) for details of these improvements.

Also, the description above is for "ideal" versions of these methods. As described, their main computational costs are for linear system solves with $\mathrm{F}$ or $\mathrm{sF}+\mathrm{G}$ (where $\mathrm{F}$ resembles a convection-diffusion operator on the velocity space and $G$ is a velocity mass matrix), $A_{p}$ or $B \widehat{G}^{-1} B^{\top}$ (pressure Poisson operators) and $G_{p}$ (a pressure mass matrix). These subsidiary systems could be solved efficiently using multigrid for $F$ or $s F+G$ and for $A_{p}$, but it is actually more effective to replace accurate solutions with approximate ones obtained, for example, by applying a single step of multigrid to the systems (see Elman et al. (2005)). In the results described below, $P_{F}$ is defined using one V-cycle of algebraic multigrid (AMG) (implemented in the IFISS software package, see Elman et al. (2007)); the pressure Poisson solves are also replaced by a single V-cycle of AMG. ${ }^{1}$ The pressure mass matrix is replaced by its diagonal approximation.

We investigate the utility of these preconditioning strategies for solving (2.2) and (2.5). The iterative linear solver we use for the preconditioned systems is GMRES. Recall from section 2 that we need to solve an instance of (2.2) in each iteration of both Algorithms 2.1 and 2.2 and an additional instance of (2.5) in each iteration of Algorithm 2.2. The right-hand side $b$ in (2.2) and (2.5) and the shift in (2.5) vary from one iteration to another. In this subsection, as a simple first test, we take $b$ to be a constant vector with unit norm whose entries all equal to $\mathrm{n}^{-0.5}$ and consider five representative values of the shift, i.e., $s=10^{-3}, 10^{-2}, 10^{-1}, 1,10$. The stopping criteria for GMRES are

$$
\|\mathbf{A} x-b\|_{2}<10^{-10} \cdot\|\mathrm{b}\|_{2}
$$

for (2.2) and

$$
\|(\mathbf{M}-\mathrm{s} \mathbf{A}) x-\mathrm{b}\|_{2}<10^{-10} \cdot\|\mathrm{b}\|_{2}
$$

for (2.5).

The following four examples are considered in this section, where (3.1) is discretized using $\mathrm{Q}_{2}-\mathrm{Q}_{1}$ mixed finite elements:

- 2D flow over an obstacle $(32 \times 128$ mesh, $\mathrm{n}=9512)$ at $\mathcal{R E}=200$ and $\mathcal{R E}=350$, and

- 2D driven-cavity flow $(64 \times 64$ mesh, $\mathrm{n}=9539)$ at $\mathcal{R E}=2000$ and $\mathcal{R E}=4000$.

Here, $\mathcal{R E}$ denotes the Reynolds number $\frac{\mathrm{UL}}{\mathrm{v}}$, where $\mathrm{U}$ is the maximum magnitude of velocity on the inflow and $\mathrm{L}$ is a characteristic length scale for the domain. (In these tests, $\mathrm{L}=2$ and $\mathrm{U}=1$ for the obstacle problem, so that $\mathcal{R E}=\frac{2}{v}$, and $\mathrm{L}=1, \mathrm{U}=1, \mathcal{R E}=\frac{1}{v}$ for the cavity problem.) In all four examples, $n \approx 10,000$. Stability analysis of these two flows has been considered in previous work (Elman et al. (2012); Elman \& $\mathrm{Wu}(2012)$ ), and their streamlines are depicted in Figure 1. The critical Reynolds number is about 370 for the flow over an obstacle and approximately 8000 for the driven-cavity flow (see Elman et al. (2012)).

\footnotetext{
${ }^{1}$ On the velocity space, AMG is actually applied to the system with coefficient matrix $\widehat{F}$ or $s \widehat{F}+G$, where $\widehat{F}$ is the block convectiondiffusion operator that would be obtained from a Picard linearization of the Navier-Stokes operator; see Elman et al. (2005) for details.
} 


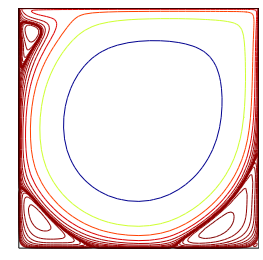

FIG. 1: The streamlines of driven-cavity flow (at $\mathcal{R} \mathcal{E}=4000$ ) and flow over an obstacle (at $\mathcal{R} \mathcal{E}=350$ )

Figures 2 and 3 display the performance of the two preconditioners for solving (2.2) and (2.5) arising from these four examples. In each subplot, the residual norms $\|\mathbf{A} x-b\|_{2}$ and $\|(\mathbf{M}-s \mathbf{A}) x-b\|_{2}$ are plotted against the number of preconditioned GMRES steps. The number next to each curve indicates the value of the shift $s$. The curves labeled with " $\infty$ " correspond to (2.2) since formally, (2.2) can be viewed as (2.5) with $s=\infty$.

The performance of GMRES for these representative shifts shows that the smaller the shift $s$ is, the easier it is to solve (2.5). This is because the smaller $s$ is, the better both preconditioners (3.4) and (3.5) approximate the Schur complement $-(\eta-s)^{2} B(s F+G)^{-1} B^{\top}$. The number of GMRES steps needed by (2.2) shows the limit of how expensive solving (2.5) can get as $s$ increases. It can also be seen that the performance of LSC is almost always stronger than that of PCD in these tests, and in the sequel we restrict our attention to LSC. The conclusions reached below for LSC would also apply in a qualitative sense to PCD.

From Figures 2 and 3, we can also observe that as the Reynolds number grows, when either preconditioner is used, (2.2) and (2.5) with a large shift become increasingly difficult to solve.

\subsection{Lyapunov solvers with iterative linear solves}

We next consider the performance of the full Lyapunov solvers when the linear solution methods described in section 3.1 are integrated into the implementation.

We again consider the following four examples: flow over an obstacle at $\mathcal{R E}=200$ and $\mathcal{R E}=350$ and driven-cavity flow at $\mathcal{R} \mathcal{E}=2000$ and $\mathcal{R E}=4000$. The stopping criteria are

$$
\|\mathrm{R}\|_{\mathrm{F}}<10^{-6} \cdot\|\mathrm{C}\|_{\mathrm{F}}
$$

for the Lyapunov solve (outer iteration) and (3.6), (3.7) for the linear solves (inner iteration). All the linear systems arising from the Lyapunov solvers will be solved using GMRES in conjunction with the LSC preconditioner described in section 3.1. In addition, as in the previous numerical experiments, the subsidiary linear systems arising from the application of $\mathbf{P}^{-1}$ to a vector are solved approximately using one multigrid V-cycle.

We first consider RKSM, which is shown in Algorithm 2.2. Recall that one solve of (2.2) and one solve of (2.5) are needed at each iteration. The results of Algorithm 2.2 are shown in Figures 4 and 5, in which we plot both the number of GMRES steps (inner iterations) and the shift $s$ for each iteration of RKSM (outer iteration).

In each of Figures $4 a, 4 c, 5 a$ and $5 c$, there are two curves representing respectively the number of GMRES steps needed for solving (2.2) (denoted by ' $\times$ ') and that for (2.5) (denoted by ' $\circ$ ') as Algorithm 2.2 proceeds. As can be seen from these four figures, the number of GMRES steps needed for (2.2) is nearly a 


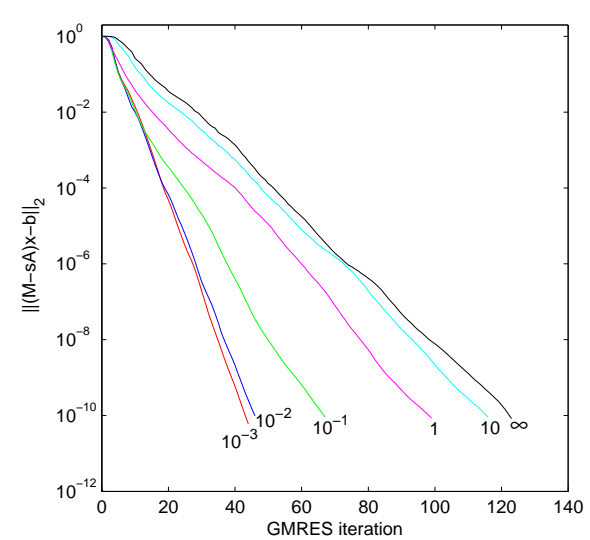

(a) PCD (Obstacle, $\mathcal{R E}=200$ )

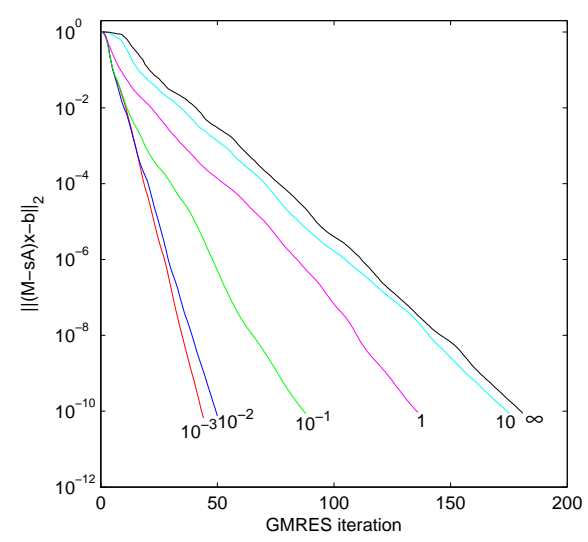

(c) PCD (Obstacle, $\mathcal{R E}=350)$

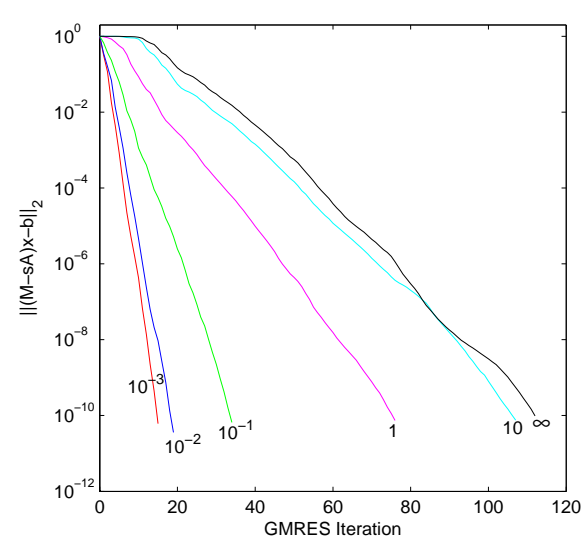

(b) LSC (Obstacle, $\mathcal{R E}=200$ )

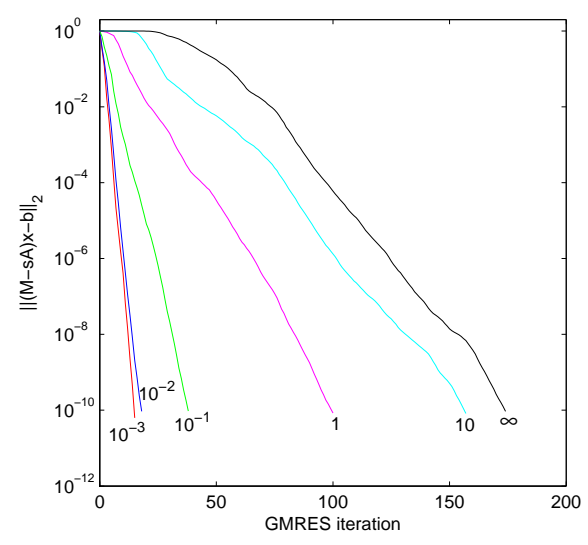

(d) LSC (Obstacle, $\mathcal{R E}=350)$

FIG. 2: The performance of the pressure convection-diffusion (PCD) preconditioner and the least-squares commutator (LSC) preconditioner in flow over an obstacle

constant as the outer iteration advances. This constant is about 110 for $\mathcal{R} \mathcal{E}=200,170$ for $\mathcal{R E}=350,360$ for $\mathcal{R E}=2000$ and 560 for $\mathcal{R E}=4000$, as given by the rightmost curve in Figures $2 \mathrm{~b}, 2 \mathrm{~d}, 3 \mathrm{~b}$ and $3 \mathrm{~d}$. On the other hand, from the same set of figures, we can see that the number of GMRES steps required to solve (2.5) is quite oscillatory and can change drastically from one iteration to the next. The pattern of oscillation matches perfectly with that of the shift, which is depicted in Figures $4 \mathrm{~b}, 4 \mathrm{~d}, 5 \mathrm{~b}$ and $5 \mathrm{~d}$ (denoted by ' $\square$ '). The bigger the shift is, the more GMRES steps are needed to solve (2.5). This behavior is again expected from the numerical experiments in the previous section. In many outer iterations, the number of GMRES steps required to solve (2.5) is significantly smaller than that needed by (2.2). In fact, in all four cases considered here, approximately $75 \%$ of all the GMRES steps taken to solve (1.4) are devoted to the solution of (2.2).

Next, we perform a similar test for the standard Krylov subspace method (Algorithm 2.1). Recall from section 2 that one solve of (2.2) is needed at each iteration of Algorithm 2.1. We continue to use (3.6) as 


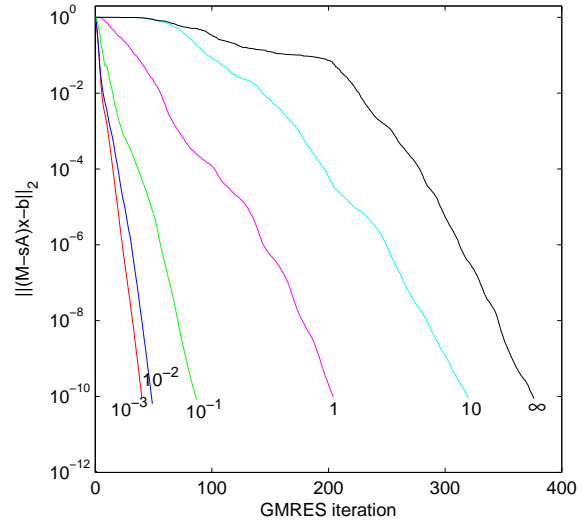

(a) PCD (Cavity, $\mathcal{R E}=2000$ )

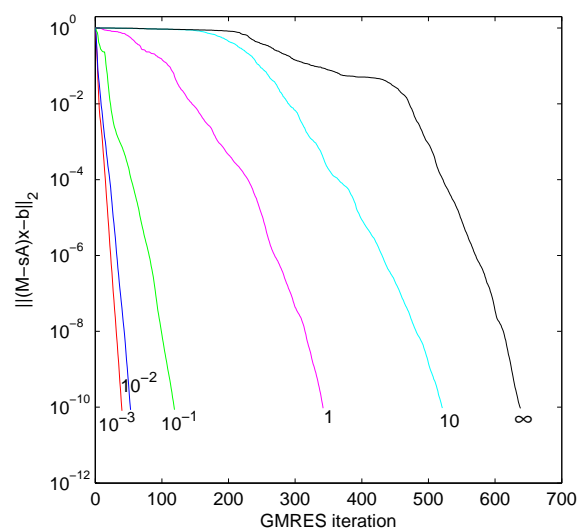

(c) $\operatorname{PCD}($ Cavity, $\mathcal{R E}=4000)$

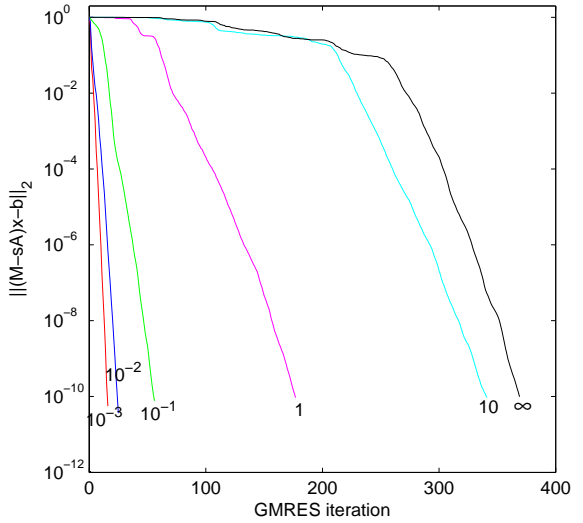

(b) LSC (Cavity, $\mathcal{R E}=2000$ )

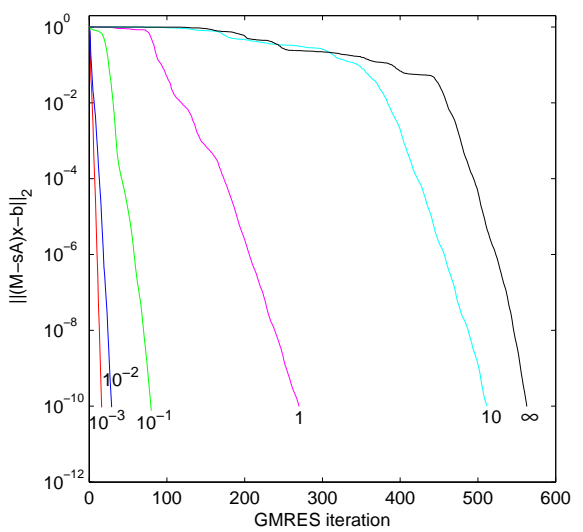

(d) LSC (Cavity, $\mathcal{R E}=4000$ )

FIG. 3: The performance of the pressure convection-diffusion (PCD) preconditioner and the least-squares commutator (LSC) preconditioner in driven-cavity flow

the stopping criterion for the linear solve (2.2) and (3.8) as the stopping criterion for the Lyapunov solve. The results of Algorithm 2.1 applied to the same set of examples are shown in Figure 6, in which each curve corresponds to one example and displays the number of GMRES iterations required to solve (2.2). This figure shows that the number of GMRES steps needed to solve (2.2) is roughly the same in each iteration of Algorithm 2.1, as observed for RKSM in Figures 4 and 5. Moreover, comparison between Figure 6 and Figures 4, 5 shows that to solve (1.4) to the same order of accuracy, the standard Krylov subspace method needs a subspace more than twice as large as that needed by RKSM.

In Figure 7, we plot the residual norm $\|R\|_{F}$ associated with (1.4) against the total number of GMRES steps required by both Lyapunov solvers. As displayed in Figure 7, in all four examples we consider, to compute an approximate solution of (1.4) that satisfies (3.8), RKSM is much more efficient, requiring approximately half as many GMRES steps as the standard Krylov subspace method. There are two reasons 


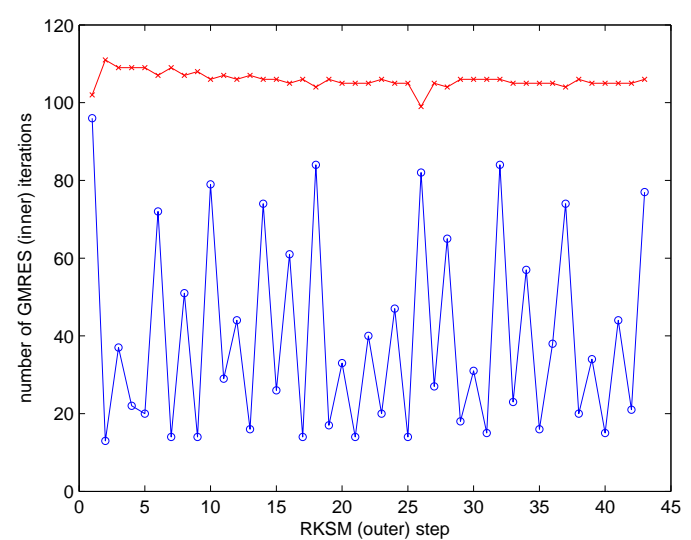

(a) inner iteration counts (Obstacle, $\mathcal{R} \mathcal{E}=200$ )

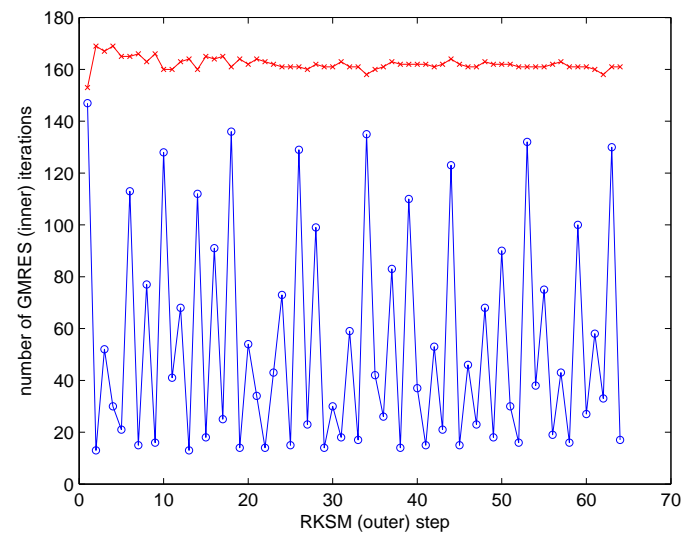

(c) inner iteration counts (Obstacle, $\mathcal{R} \mathcal{E}=350$ )

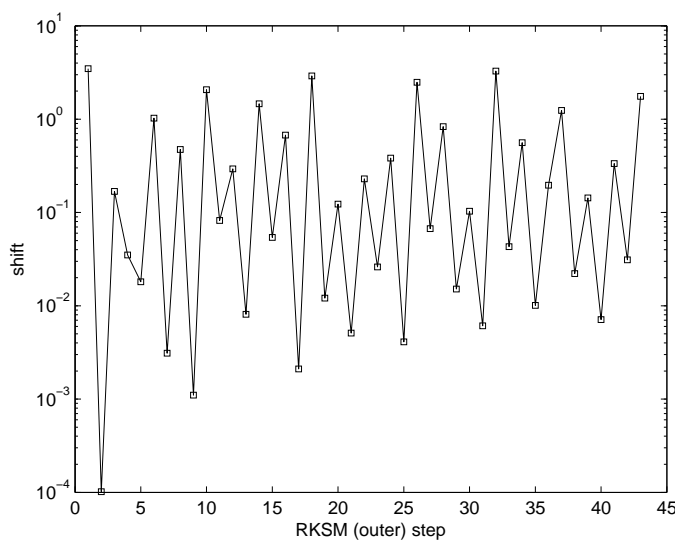

(b) shifts (Obstacle, $\mathcal{R} \mathcal{E}=200$ )

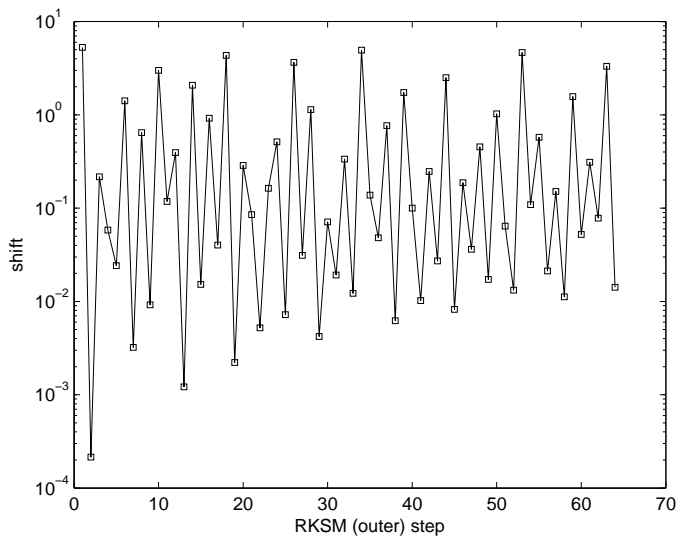

(d) shifts (Obstacle, $\mathcal{R E}=350$ )

FIG. 4: Inner iteration counts and shifts of RKSM applied to flow over an obstacle $(\times$ : the number of GMRES steps needed for solving (2.2), ○: the number of GMRES steps needed for solving (2.5), $\square$ : the shift in (2.5))

for this: first, RKSM requires a much smaller Krylov subspace, and second, although an extra solve of (2.5) is needed per iteration of RKSM, it is on average much cheaper to solve this problem than to solve (2.2).

\section{Modified rational Krylov subspace method}

As shown in section 3, the cost of RKSM applied to (1.4) is dominated by that of solving the linear system (2.2). If the solution of (2.2) can somehow be avoided without harming the convergence rate of RKSM, then the efficiency of this method will increase significantly. In this section, we propose a modified version of RKSM for (1.4) that achieves this goal.

Recall from section 2 that when RKSM is applied to (1.4), the linear system (2.5) arises from computing 


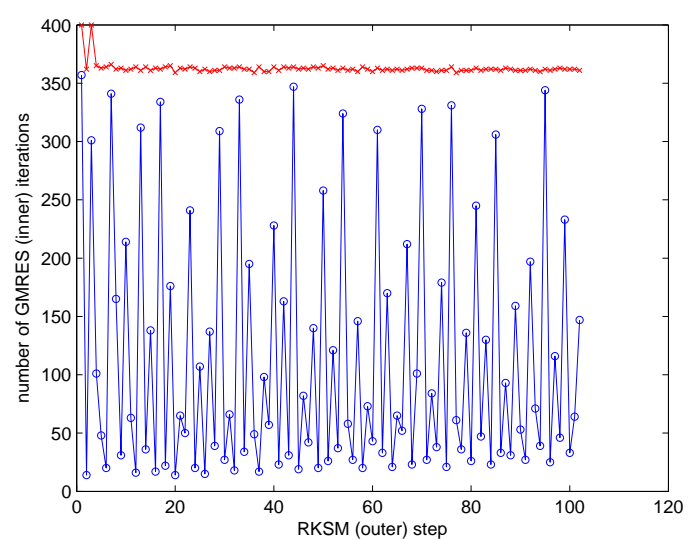

(a) inner iteration counts (Cavity, $\mathcal{R E}=2000$ )

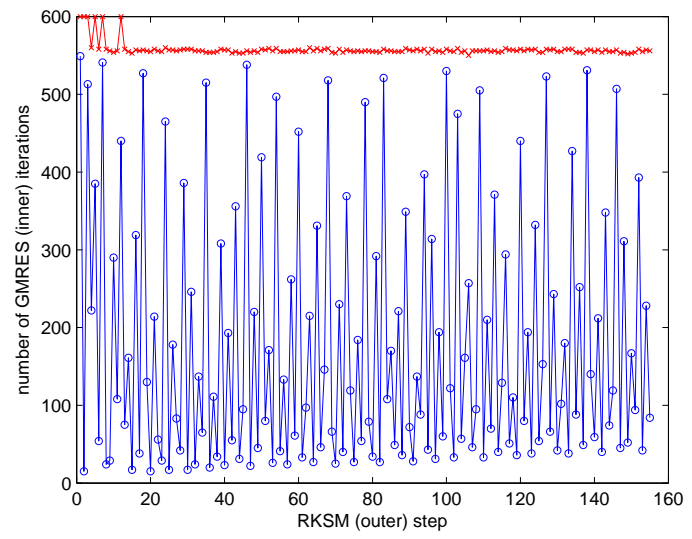

(c) inner iteration counts (Cavity, $\mathcal{R} \mathcal{E}=4000$ )

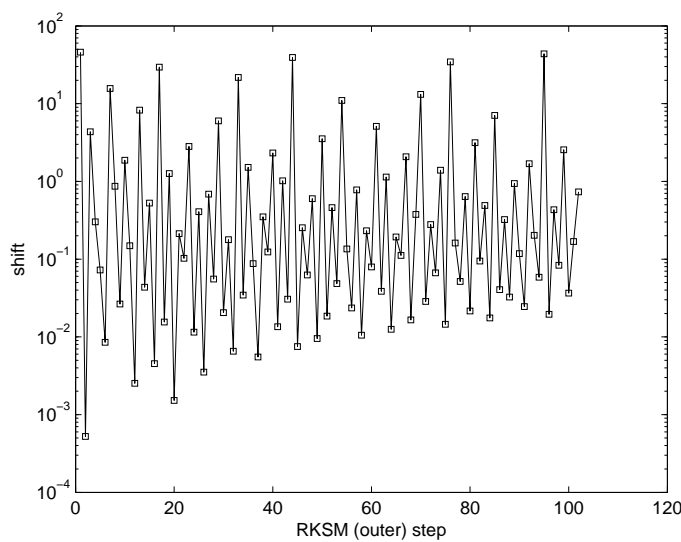

(b) shifts (Cavity, $\mathcal{R} \mathcal{E}=2000$ )

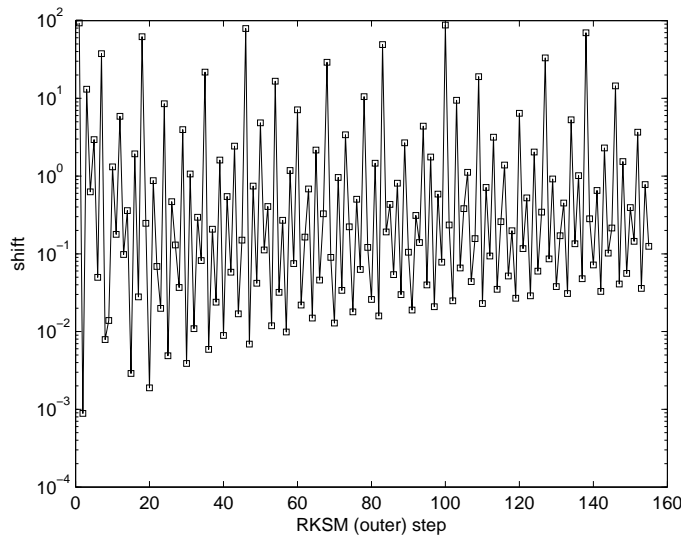

(d) shifts (Cavity, $\mathcal{R} \mathcal{E}=4000$ )

FIG. 5: Inner iteration counts and shifts of RKSM applied to driven-cavity flow ( $\times$ : the number of GMRES steps needed for solving (2.2), ○: the number of GMRES steps needed for solving (2.5), $\square$ : the shift in (2.5))

a new Krylov vector and the linear system (2.2) arises from the computation of the matrix $T_{m}=\mathbf{V}^{\top} S \mathbf{V}$. $T_{m}$ is needed in the construction of the approximate solution $V_{m} X_{m} V_{m}^{\top}$ of (1.4), and its eigenvalues are used to generate the next shift $s_{m+1}$. However, it is not necessary to compute an approximate solution to (1.4) at each step of Algorithm 2.2; moreover, we only need the eigenvalues of $T_{m}$ in (2.3), not $T_{m}$ itself.

Thus, we can reduce the number of solves by not computing $T_{m}$ at every step. We propose using the eigenvalues of $\mathcal{T}_{\mathfrak{m}}=\left(V_{m}^{\top} \mathbf{A} V_{m}\right)^{-1}\left(V_{m}^{\top} \mathbf{M} V_{m}\right)$ in (2.3) to generate the shift, instead of those of $T_{m}$. The reason is twofold: first, constructing $\mathcal{T}_{\mathfrak{m}}$ only requires matrix-vector products with $\mathbf{A}$ and $\mathbf{M}$; second, the eigenvalues of $\mathcal{T}_{m}$ approximate those of $T_{m}$ well. The latter assertion is supported by numerical evidence given below and in section 5 .

Consider again (1.4) arising from driven-cavity flow at $\mathcal{R E}=2000$. We compute the eigenvalues of both 


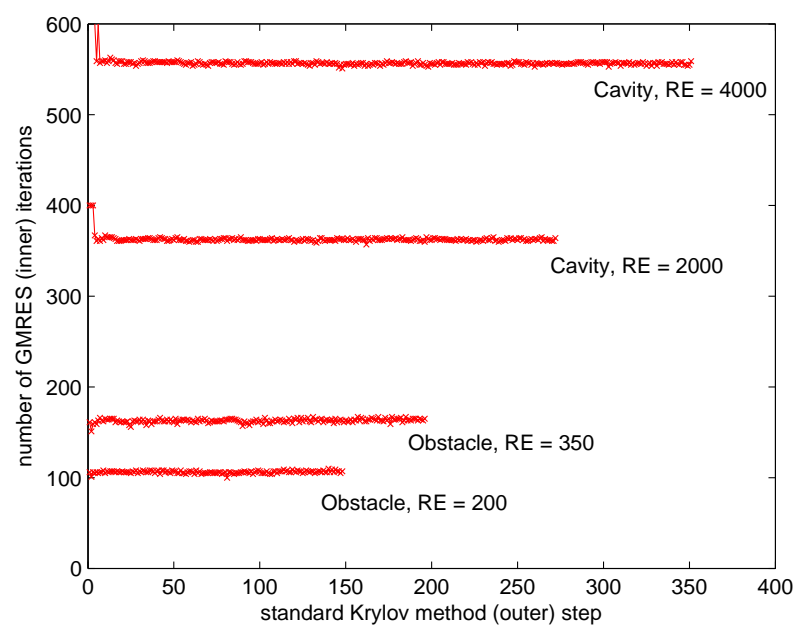

FIG. 6: Inner iteration counts of the standard Krylov subspace method applied to all four examples $(\times$ : the number of GMRES steps needed for solving (2.2))

$T_{m}$ and $\mathcal{T}_{m}$ as Algorithm 2.2 proceeds. For $m=25,50,75,100$, the spectra of $T_{m}$ and $\mathcal{T}_{m}$ are plotted in Figure 8, in which the crosses denote the eigenvalues of $T_{m}$ and the circles represent the eigenvalues of $\mathcal{T}_{m}$. (Note that a logarithmic scale is used on the real axis for a clearer display of the eigenvalues.) As shown in Figure 8, the eigenvalues of $\mathcal{T}_{m}$ indeed approximate those of $T_{m}$ well, especially for larger $m$. This suggests that replacing the eigenvalues of $T_{m}$ with those of $\mathcal{T}_{m}$ in the computation of the new shift will not affect the asymptotic convergence rate of RKSM.

The variant of RKSM with this modification is outlined in Algorithm 4.1. In the modified algorithm, we compute $T_{m}$ and check the convergence of the Lyapunov solve only when the iteration count $m$ is an integer multiple of a prescribed integer $k$. (When $k=1$, this is simply Algorithm 2.2.) Consequently, (2.2) appears only in those iterations. In the iterations where $T_{m}$ is not computed, we continue using the approach proposed in Druskin \& Simoncini (2011) to choose the next shift; the only change is to use the eigenvalues of $\mathcal{T}_{\mathfrak{m}}$ instead of those of $T_{m}$ in (2.3). The computation of $\mathcal{T}_{m}$ entails only two matrix-vector products $\mathbf{A} v_{m}$ and $\mathbf{M} v_{m}$ at each iteration. In fact, only the matrix-vector product $\mathbf{M} v_{m}$ is needed since according to (2.4), $\mathbf{A} v_{\mathfrak{m}}$ is the right-hand side of (2.5) and has to be computed anyway. Thus, the cost of constructing $\mathcal{T}_{\mathfrak{m}}$ is negligible.

Algorithm 4.1 The modified rational Krylov subspace method for (1.4)

1. Given a tolerance $\tau$, a shift $s_{1}$ and an integer $k>1$. Let $v_{1}=V_{1}=P$.

2. For $m=1,2, \cdots$

$$
\begin{gathered}
\text { 2.1. } w=\left(S-s_{m} I\right)^{-1} v_{m} . \\
\text { For } i=1, \ldots, m \\
h_{i, m} \leftarrow v_{i}^{\top} w ; \\
w \leftarrow w-v_{i} h_{i, m} .
\end{gathered}
$$

2.2. Compute the reduced QR factorization of $w: w=v_{m+1} h_{m+1, m}$.

\subsection{If $\bmod (\mathbf{m}, \mathbf{k})=\mathbf{0}$}

2.3.1. compute $T_{m}=V_{m}^{\top} S V_{m}$ and solve the small Lyapunov equation 


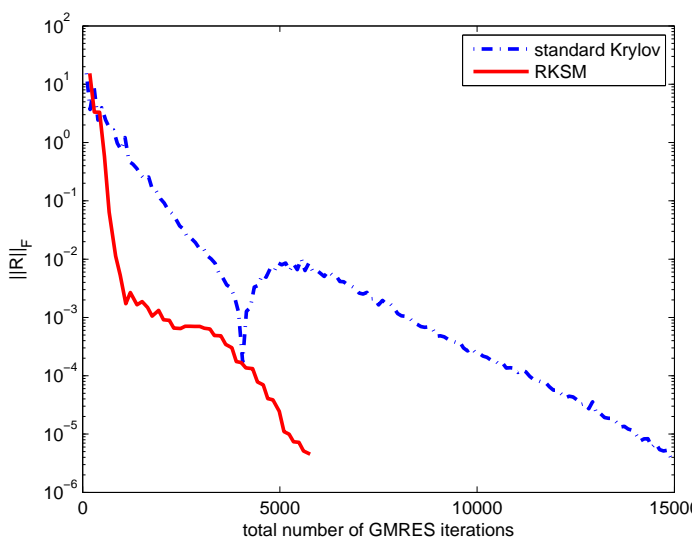

(a) Obstacle, $\mathcal{R E}=200$

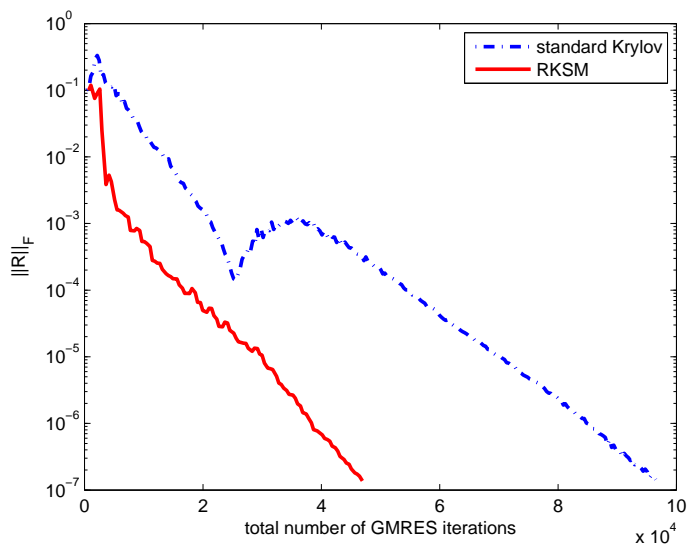

(c) Cavity, $\mathcal{R E}=2000$

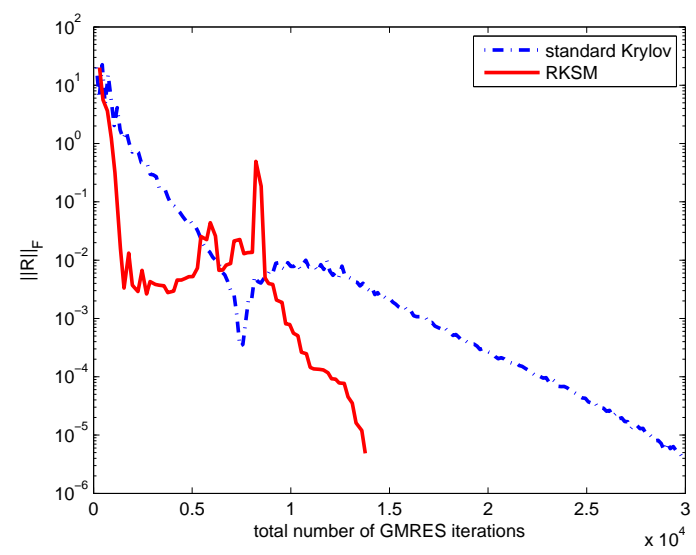

(b) Obstacle, $\mathcal{R} \mathcal{E}=350$

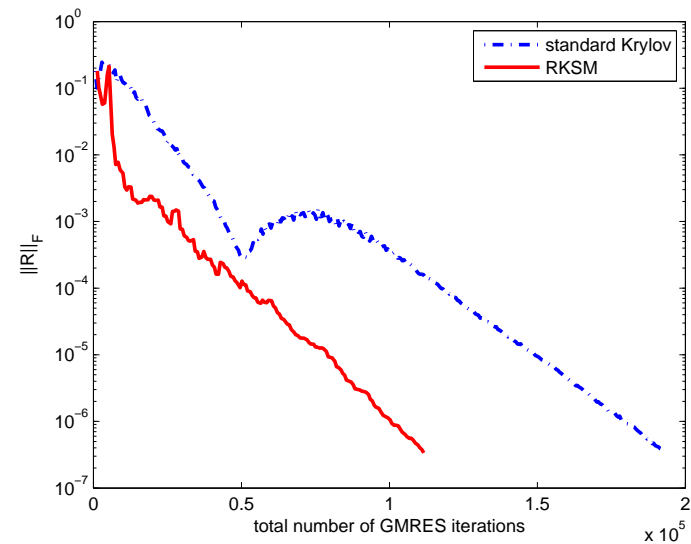

(d) Cavity, $\mathcal{R E}=4000$

FIG. 7: Comparison of the total number of GMRES iterations required by the standard Krylov subspace method and RKSM

$$
T_{m} X_{m}+X_{m} T_{m}^{\top}=\left(V_{m}^{\top} P\right) C\left(V_{m}^{\top} P\right)^{\top} ;
$$

2.3.2. if $\|\mathrm{R}\|_{\mathrm{F}}<\tau$, then stop.

2.4. Else, compute $\mathcal{T}_{m}=\left(V_{m}^{\top} \mathbf{A} V_{m}\right)^{-1}\left(V_{m}^{\top} \mathbf{M} V_{m}\right)$.

2.5. $\mathrm{V}_{\mathrm{m}+1} \leftarrow\left[\mathrm{V}_{\mathrm{m}}, v_{\mathrm{m}+1}\right]$ and compute the next shift $\mathrm{s}_{\mathrm{m}+1}$.

We apply Algorithm 4.1 (with $k=5$ ) to the four examples considered in the previous section and display the numerical results in Figure 9. We continue to use the stopping criteria (3.6), (3.7) for the inner iterations and (3.8) for the outer iterations. In Figure 9, the crosses and the circles again denote the numbers of preconditioned GMRES steps required to solve (2.2) and (2.5), respectively. Since we only compute $S v_{m+1}$ 


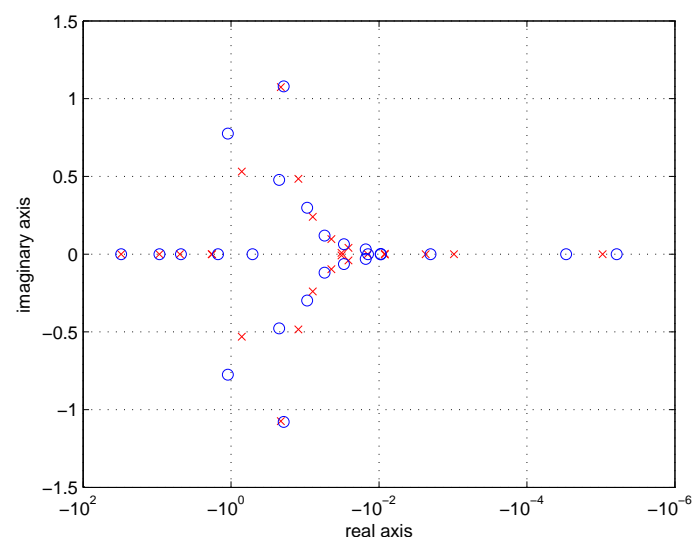

(a) $m=25$

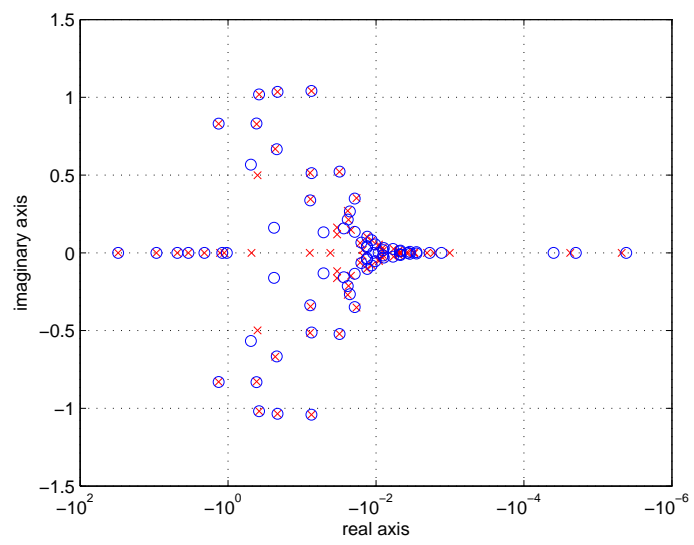

(c) $\mathrm{m}=75$

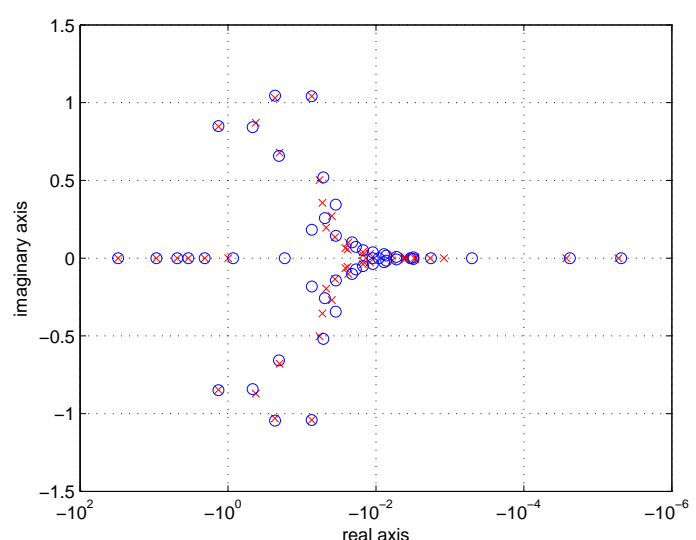

(b) $m=50$

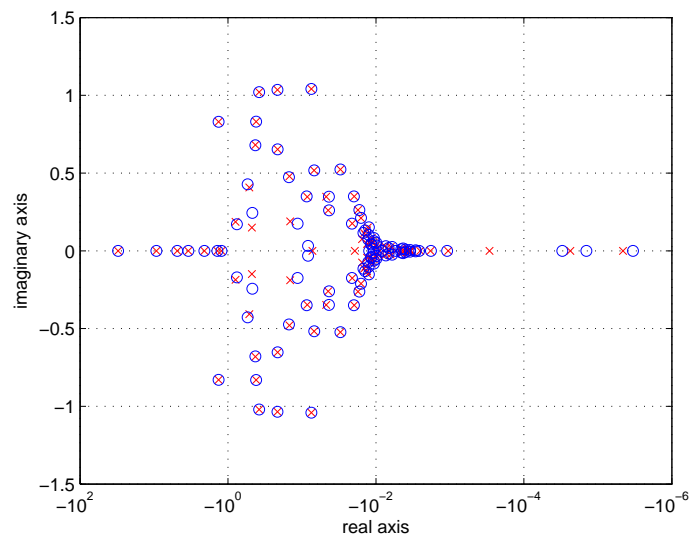

(d) $m=100$

FIG. 8: The eigenvalues of $T_{m}$ (crosses) and $\mathcal{T}_{m}$ (circles)

every $k=5$ iterations, as seen in Figure 9, the number of GMRES iterations taken to solve (2.2) is simply zero in many iterations of Algorithm 4.1. By comparing Figures 4, 5 and 9, we also observe that for the same example, the sizes of the Krylov subspaces built by Algorithm 2.2 and that built by Algorithm 4.1 are almost the same. (A slight increase (less than $\mathrm{k}$ ) in the size of the Krylov subspace can be observed when Algorithm 4.1 is used instead of Algorithm 2.2. This is due to the fact that in Algorithm 4.1, we only check convergence every $k$ iterations and, therefore, the algorithm may not terminate even if (3.8) has already been met.) This implies that the shifts generated using the eigenvalues of $\mathcal{T}_{\mathfrak{m}}$ are essentially of the same quality as those generated using the eigenvalues of $\mathrm{T}_{\mathrm{m}}$.

We also compare the total number of GMRES steps required by Algorithms 2.1, 2.2 and 4.1 (with $k=5$ or $k=10$ ) for solving (1.4). In Figure 10, the residual norm of (1.4) is again plotted against the total number of GMRES steps needed by the Lyapunov solvers. These residual curves show that Algorithm 4.1 converges much more rapidly than Algorithm 2.2. In order to produce an approximate solution that satisfies (3.8), 
Algorithm 4.1 (with $k=5$ ) takes about 50\% fewer GMRES steps. We can further improve the efficiency of Algorithm 4.1 by increasing $k$ to 10, though as shown in Figure 10, such improvement is much less pronounced. This is because when $k=5$, the dominant cost of Algorithm 4.1 has already become the solution of (2.5), which cannot be made any cheaper by increasing $\mathrm{k}$.

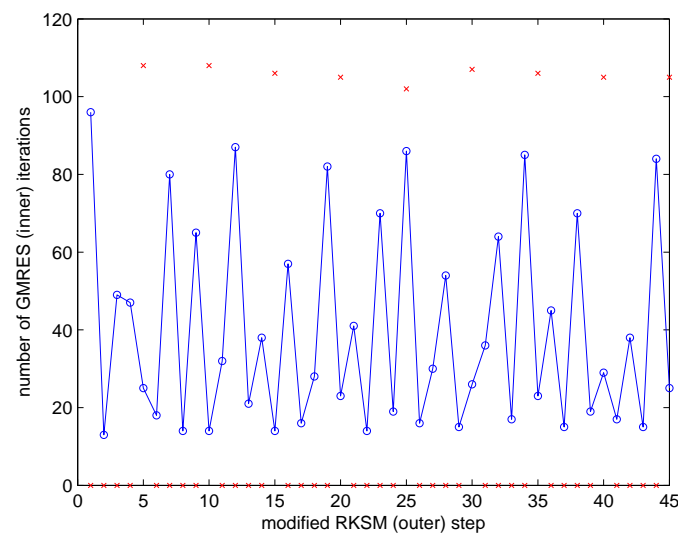

(a) Obstacle, $\mathcal{R E}=200$

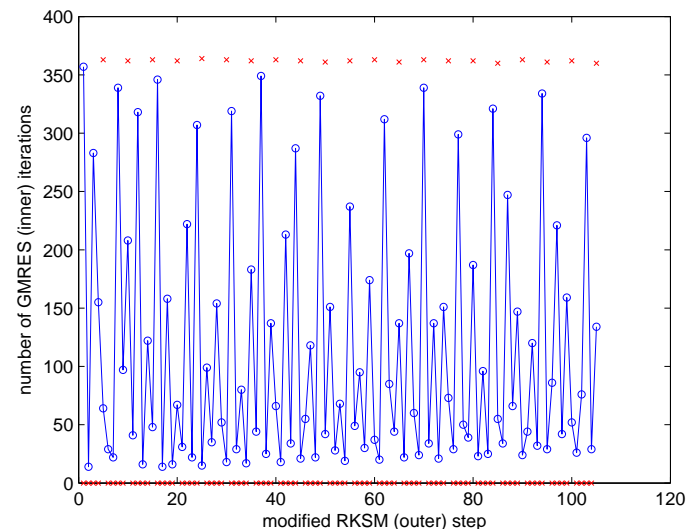

(c) Cavity, $\mathcal{R E}=2000$

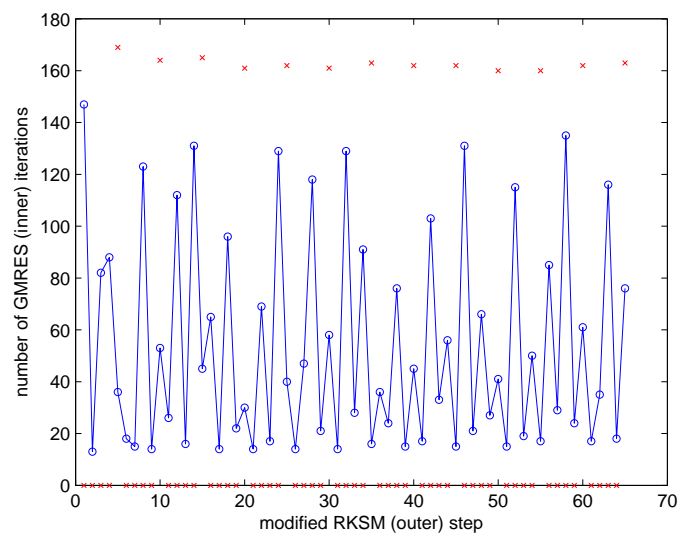

(b) Obstacle, $\mathcal{R E}=350$

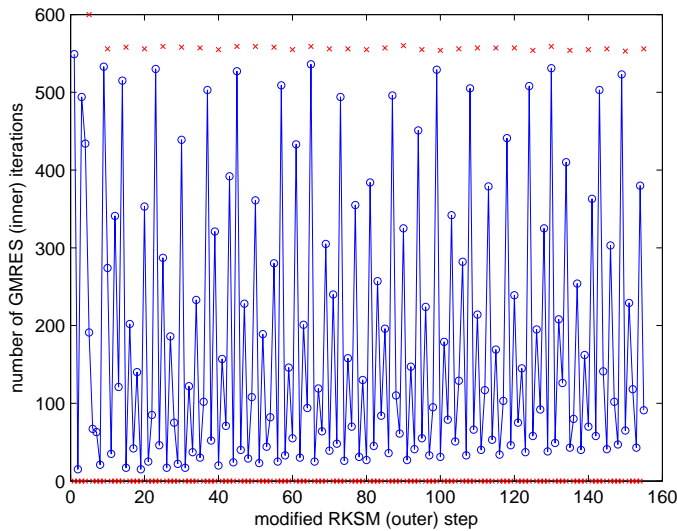

(d) Cavity, $\mathcal{R E}=4000$

FIG. 9: Inner iteration counts of the modified RKSM with $k=5$

\section{Further analysis of $T_{m}$ and $\mathcal{T}_{m}$}

The modified RKSM (Algorithm 4.1) proposed in section 4 is based on the hypothesis that the eigenvalues of $\mathcal{T}_{m}=\left(V_{m}^{\top} \mathbf{A} V_{m}\right)^{-1}\left(V_{m}^{\top} \mathbf{M} V_{m}\right)$ approximate those of $T_{m}=V_{m}^{\top} S V_{m}=V_{m}^{\top}\left(\mathbf{A}^{-1} \mathbf{M}\right) V_{m}$ well. In this section, we analyze the relation between $\mathcal{T}_{m}$ and $T_{m}$ and provide more numerical evidence to support this hypothesis.

As shown in Ruhe (1994) (also see the proof of Proposition 4.2 of Druskin \& Simoncini (2011)), the 


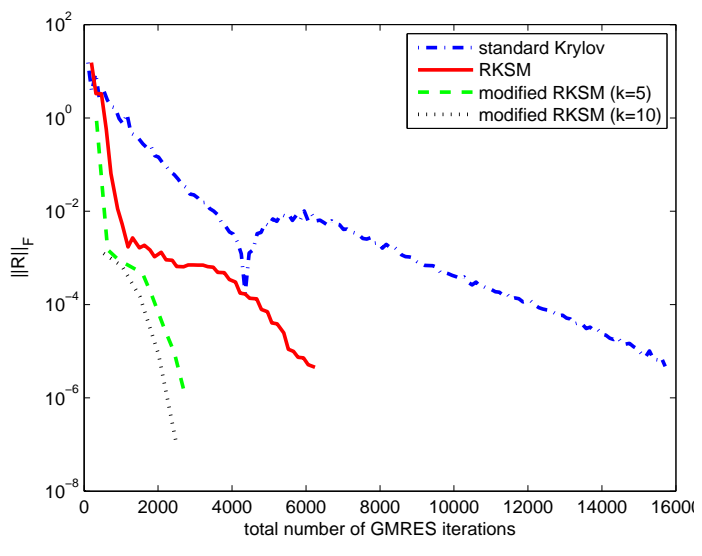

(a) Obstacle, $\mathcal{R} \mathcal{E}=200$

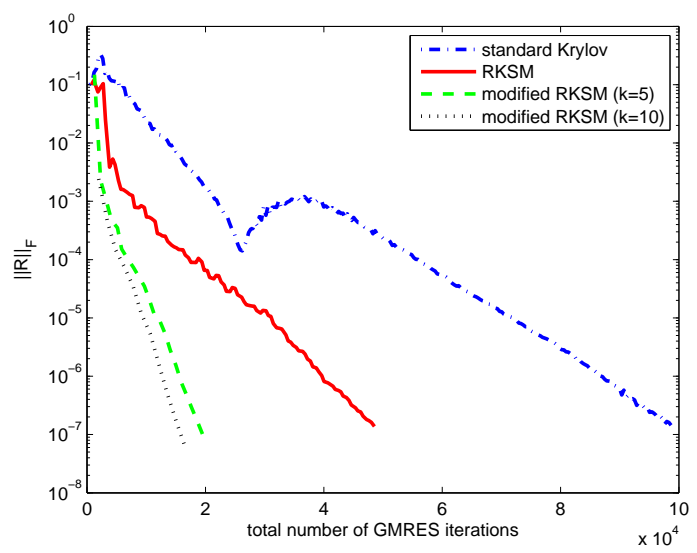

(c) Cavity, $\mathcal{R E}=2000$

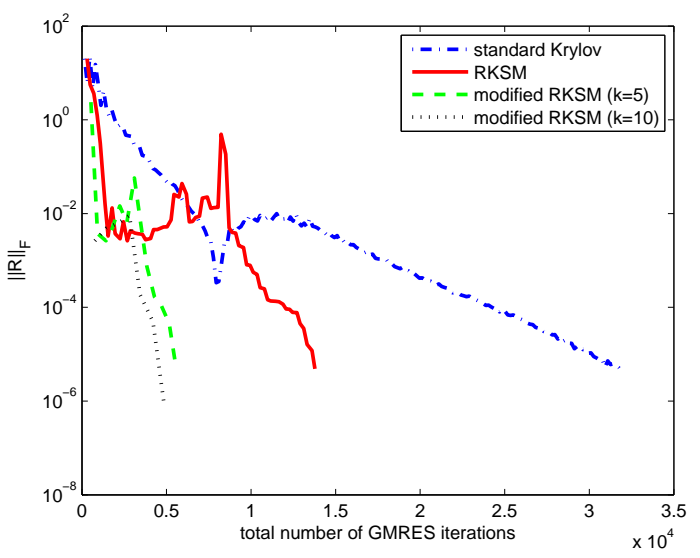

(b) Obstacle, $\mathcal{R} \mathcal{E}=350$

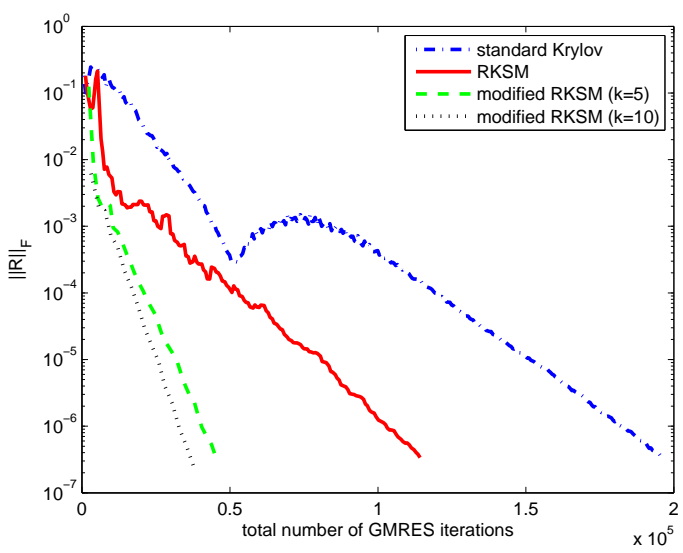

(d) Cavity, $\mathcal{R E}=4000$

FIG. 10: Comparison of the total number of GMRES iterations required by the standard Krylov subspace method, RKSM and modified RKSM

rational Krylov subspace method computes the Arnoldi decomposition

$$
S V_{m}=V_{m} T_{m}+v_{m+1} h_{m+1, m} e_{m}^{\top} D_{m} H_{m}^{-1}-\left(I-V_{m} V_{m}^{\top}\right) S v_{m+1} h_{m+1, m} e_{m}^{\top} H_{m}^{-1}
$$

at each iteration, where $e_{m}$ is the last column of the $m \times m$ identity matrix, $D_{m}=\operatorname{diag}\left(\left\{s_{1}, s_{2}, \ldots, s_{m}\right\}\right)$ is the diagonal matrix that holds all the previous shifts, and $I$ is the $n \times n$ identity matrix. The definitions of $V_{m}, H_{m}, T_{m}, h_{m+1, m}$ and $v_{m+1}$ can be found in Algorithm 2.2.

THEOREM 5.1 $\mathcal{T}_{m}=T_{m}+E_{m}$ where $E_{m}$ has $m-1$ zeros eigenvalues. The single nonzero eigenvalue of $E_{m}$ is

$$
\rho_{m}=h_{m+1, m} e_{m}^{\top} H_{m}^{-1}\left(V_{m}^{\top} A V_{m}\right)^{-1} V_{m}^{\top} \mathbf{A}\left(I-V_{m} V_{m}^{\top}\right)\left(s_{m} I-S\right) v_{m+1}
$$


and the corresponding eigenvector is

$$
\psi_{m}=\left(V_{m}^{\top} \mathbf{A} V_{m}\right)^{-1} V_{m}^{\top} \mathbf{A}\left(I-V_{m} V_{m}^{\top}\right)\left(s_{m} I-S\right) v_{m+1}
$$

Proof. Left multiply both sides of (5.1) by $\mathrm{V}_{\mathrm{m}}^{\top} \mathbf{A}$ :

$$
\begin{aligned}
V_{m}^{\top} \mathbf{M} V_{m}= & \left(V_{m}^{\top} \mathbf{A} V_{m}\right) T_{m}+V_{m}^{\top} A v_{m+1} h_{m+1, m} e_{m}^{\top} D_{m} H_{m}^{-1} \\
& -V_{m}^{\top} \mathbf{A}\left(I-V_{m} V_{m}^{\top}\right) S v_{m+1} h_{m+1, m} e_{m}^{\top} H_{m}^{-1}
\end{aligned}
$$

Then left multiply both sides of (5.2) by $\left(\mathrm{V}_{\mathrm{m}}^{\top} \mathbf{A} \mathrm{V}_{\mathrm{m}}\right)^{-1}$ :

$$
\begin{aligned}
\mathcal{T}_{m}= & T_{m}+\left(V_{m}^{\top} \mathbf{A} V_{m}\right)^{-1} V_{m}^{\top} \mathbf{A} v_{m+1} h_{m+1, m} e_{m}^{\top} D_{m} H_{m}^{-1} \\
& -\left(V_{m}^{\top} \mathbf{A} V_{m}\right)^{-1} V_{m}^{\top} \mathbf{A}\left(I-V_{m} V_{m}^{\top}\right) S v_{m+1} h_{m+1, m} e_{m}^{\top} H_{m}^{-1}
\end{aligned}
$$

Since $e_{m}^{T} D_{m}=s_{m} e_{m}^{T}$ and $v_{m+1}$ is orthogonal to the columns of $V_{m}$, the difference between $\mathcal{T}_{m}$ and $T_{m}$ is

$$
E_{m}=h_{m+1, m}\left(V_{m}^{\top} \mathbf{A} V_{m}\right)^{-1} V_{m}^{\top} \mathbf{A}\left(I-V_{m} V_{m}^{\top}\right)\left(s_{m} I-S\right) v_{m+1} e_{m}^{\top} H_{m}^{-1}
$$

It is easy to check that $E_{m}$ has $m-1$ zero eigenvalues whose eigenvectors are given by $\left\{\psi_{j}=H_{m} e_{j}\right\}_{j=1}^{m-1}$, and the single nonzero eigenvalue $\rho_{m}$ whose corresponding eigenvector is $\psi_{m}$.

Thus, $\mathcal{T}_{m}$ differs from $T_{m}$ by a matrix of rank one. We have seen that the eigenvalues of theses two matrices are very close to each other. In Table 1 , we also report the "relative error" $\left\|E_{m}\right\|_{2} /\left\|T_{m}\right\|_{2}$ for several different values of $m$ and each of the four examples considered in Sections 3 and 4 . It can be seen that these errors are small, giving further indication that $\mathcal{T}_{m}$ is close to $T_{m}$.

Table 1: $\left\|\mathrm{E}_{\mathrm{m}}\right\|_{2} /\left\|\mathrm{T}_{\mathrm{m}}\right\|_{2}$

\begin{tabular}{c|c|c|c|c}
\hline $\mathrm{m}$ & $\begin{array}{c}\text { Obstacle } \\
\mathcal{R} \mathcal{E}=200\end{array}$ & $\begin{array}{c}\text { Obstacle } \\
\mathcal{R} \mathcal{E}=350\end{array}$ & $\begin{array}{c}\text { Cavity } \\
\mathcal{R} \mathcal{E}=2000\end{array}$ & $\begin{array}{c}\text { Cavity } \\
\mathcal{R} \mathcal{E}=4000\end{array}$ \\
\hline 25 & 0.25732 & 0.25663 & 0.03824 & 0.01553 \\
50 & 0.14088 & 0.10365 & 0.03453 & 0.02342 \\
75 & 0.05734 & 0.08111 & 0.02904 & 0.01784 \\
100 & 0.04143 & 0.05611 & 0.01797 & 0.02037 \\
\hline
\end{tabular}

\section{Conclusions}

In this paper, we explore the performance of standard Krylov subspace method and rational Krylov subspace method with iterative linear solves. Different preconditioners are tested and compared on the linear systems arising from the two Lyapunov solvers. These systems can be divided into two categories: ones with structure identical to those that arise in the computation of steady states of a system of PDEs, and ones with structure like those arising from transient PDEs. We observe that the cost of solving the linear systems of the first type dominates the total cost of rational Krylov subspace method. In light of this observation, we modify this method in such a way that solution of the first type of linear systems can mostly be avoided. The modification is simple yet effective, leading to significant savings in computational cost without degrading the convergence of the Lyapunov solver. 


\section{Funding}

This work was supported in part by the U. S. Department of Energy under grant DE-SC0009301 and by the U. S. National Science Foundation under grant DMS1115317.

\section{REFERENCES}

Antoulas, A. C., Sorensen, D. C. \& Zhou, Y. (2001) On the decay of Hankel singular values and related issues. Technical Report 01-09. Houston: Department of Computational and Applied Mathematics, Rice University. Available from http://www.caam.rice.edu/ sorensen/Tech_Reports.html.

Bartels, R. H. \& Stewart, G. W. (1972) Algorithm 432: solution of the matrix equation $A X+X B=C$. Comm. of the ACM, 15, 820-826.

Cliffe, K. A., Garratt, T. J. \& Spence, A. (1994) Eigenvalues of block matrices arising from problems in fluid mechanics. SIAM J. Matrix Anal. Appl., 15, 1310-1318.

Druskin, V., Lieberman, C. \& Zaslavsky, M. (2010) On adaptive choice of shifts in rational Krylov subspace reduction of evolutionary problems. SIAM J. Sci. Comput., 32, 2485-2496.

Druskin, V., Knizhnerman, L. \& Simoncini, V. (2011) Analysis of the rational Krylov subspace and ADI methods for solving the Lyapunov equation. SIAM J. Numer. Anal., 49, 1875-1898.

Druskin, V. \& SimONCINI, V. (2011) Adaptive rational Krylov subspaces for large-scale dynamical systems. Systems Control Lett., 60, 546-560.

Elman, H., Silvester, D. \& Wathen, A. (2005) Finite Elements and Fast Iterative Solvers. Oxford: Oxford University Press.

Elman, H. C. (2005) Preconditioning strategies for models of incompressible flow. J. Sci. Comput., 25, 347-366.

Elman, H. C., Ramage, A. \& Silvester, D. J. (2007) Algorithm 866: IFISS, a Matlab toolbox for modelling incompressible flow. ACM Transactions on Mathematical Software, 33, 14:1-14:18.

Elman, H. C., Meerbergen, K., Spence, A. \& WU, M. (2012) Lyapunov inverse iteration for identifying Hopf bifurcations in models of incompressible flow. SIAM J. Sci. Comput., 34, A1584-A1606.

Elman, H. C. \& Tuminaro, R. S. (2009) Boundary conditions in approximate commutator preconditioners for the Navier-Stokes equations. Electron. Trans. Numer. Anal., 35, 257-280.

Elman, H. C. \& WU, M. (2012) Lyapunov inverse iteration for computing a few rightmost eigenvalues of large generalized eigenvalue problems. Technical Report TR-5009. College Park: University of Maryland Department of Computer Science. To appear in SIAM J. Matrix Anal. Appl.

GRASEDYCK, L. (2004) Low-rank solutions of the Sylvester equation. Numer. Linear Algebra Appl., 11, 371-389.

HAmmarling, S. J. (1982) Numerical solution of the stable, non-negative definite Lyapunov equation. IMA J. Numerical. Anal., 2, 303-323.

Jaimoukha, I. M. \& Kasenally, E. M. (1994) Krylov subspace methods for solving large Lyapunov equations. SIAM J. Numer. Anal., 31, 227-251.

Kressner, D. \& Tobler, C. (2010) Low-rank tensor Krylov subspace methods for parameterized linear systems. Technical Report 2010-16. Zurich: ETH. Available from http: / /www. math. ethz . ch/ kressner/.

MeErbergen, K. \& Spence, A. (2010) Inverse iteration for purely imaginary eigenvalues with application to the detection of Hopf bifurcation in large scale problems. SIAM J. Matrix Anal. Appl., 31, 1982-1999.

Meerbergen, K. \& VAndebril, R. (2012) A reflection on the implicitly restarted Arnoldi method for computing eigenvalues near a vertical line. Linear Algebra Appl., 436, 2828-2844.

PENZL, T. (2000) Eigenvalue decay bounds for solutions of Lyapunov equations: the symmetric case. Systems Control Lett., 40, 139-144.

RuHE, A. (1984) Rational Krylov sequence methods for eigenvalue computation. Lin. Alg. Appl., 58, 391-405.

RUHE, A. (1994) The rational Krylov algorithm for nonsymmetric eignevalue problems. III: complex shifts for real matrices. BIT, 34, 165-176. 
20 of 20

SAAD, Y. (1990) Numerical solution of large Lyapunov equations. Signal Processing, Scattering, Operator Theory, and Numerical Methods (M. A. Kaashoek, J. H. van Schuppen \& A. C. Ran eds). Proceedings of the International Symposium MTN-89, vol. III. Boston: Birkhauser, pp. 503-511.

Simoncini, V. (2007) A new iterative method for solving large-scale Lyapunov matrix equations. SIAM J. Sci. Comput., 29, 1268-1288.

STEWART, G. W. (2001) Matrix Algorithms Volume II: Eigensystems. Philadelphia: SIAM. 